\title{
The Efficiency-Equality Tradeoff in Welfare State Economies
}

\author{
Radim Boháček* \\ CERGE-EI, Prague, Czech Republic
}

February 5, 2002

\begin{abstract}
This paper studies the effects of different levels of social insurance on efficiency and distribution of resources in a general equilibrium model of a closed economy with heterogeneous agents and moral hazard. I compare optimal allocations of capital, labor supply, and consumption in stationary recursive equilibria for economies with different guaranteed minimum consumption levels (social insurance). I show that the efficiency-equality tradeoff associated with welfare state economies does not hold. Efficiency decreases and equality rises as the minimal guaranteed consumption increases from zero to around one third of the average consumption. However, if social insurance expands even further, the efficiency loss becomes very high and equality worsens. Average welfare is greater in economies with high social insurance while the median agent is better off in economies with low social insurance. Finally, I study the transitions between welfare regimes' steady states to evaluate the effects of social insurance reforms.

JEL Classification: E60, C68, D82, I38.

${ }^{*}$ I am grateful to Bee Jeong, Michal Kejak, Thomas J. Sargent, Nancy L. Stokey, the members of the Money and Banking and Theory Lunch Workshops, and especially to Fernando Alvarez, Robert E. Lucas, Jr., Edward C. Prescott, Robert M. Townsend, and Carlos Pérez-Verdía for suggestions and criticism. Natalia Kovrizhnyh and Bohdan Vaněk provided excellent research assistance. This research was supported by a grant from the CERGE-EI foundation under program of the Global Development Network. All opinions expressed are those of the author and have not been endorsed by CERGE-EI or the GDN. Address: CERGE-EI, Politickych veznu 7, 11221 Prague 1, Czech Republic. Email: radim.bohacek@cerge-ei.cz.
\end{abstract}




\section{Introduction}

One of the important differences among individual countries is the degree to which governments protect citizens from idiosyncratic losses of income or well-being. For example, unemployment benefits during the first year of unemployment amount approximately to two-thirds of the average income in European countries but only to one half of the average income in the non-European OECD countries. Correspondingly, social protection expenditures aggregate to $25-40 \%$ of gross domestic product in Europe while only to 10-20\% in the United States and other OECD economies (see Table 1). The goal of this paper is to characterize the effects of different levels of social insurance on efficiency and the distribution of resources in a general equilibrium model with moral hazard.

Social insurance today is generally administered by national governments with the following "three basic aims: to prevent poverty, to provide social security in the sense of helping people to preserve their social status in the case of lost earnings, and to reduce inequalities in living conditions" Flora (1986). ${ }^{1}$ Considering the large differences in social insurance among individual countries, there seems to be a widespread notion of the "welfare state tradeoff" between efficiency and equality. It usually compares the United States to Europe with the following "common sense" conclusions: 1) European countries provide generous social insurance that prevents individual citizens from falling into poverty; 2) redistribution of resources by European governments generates a relatively equal distribution of income; 3) European social insurance is costly in terms of economic efficiency; but 4) the European welfare state provides better quality of life despite the efficiency loss.

This paper is to my knowledge the first one that addresses these issues in a dynamic, general equilibrium model with insurance and moral hazard. The moral hazard-modelled as unobserved labor effort in production technology - captures the incentive burden associated with the welfare state policies. ${ }^{2}$ The model abstracts from other important phenomena usually associated with welfare state economies: progressive taxation for redistribution of income, budget deficits related to funding of welfare state redistributions and benefits, large public sector and 
public spending, wide marginal tax wedges, detailed regulations and cartelization (especially in the labor market), and an inflation-prone system of wage formation.

In order to evaluate the effects of social insurance on distribution of resources, I consider steady states of economies with a stationary distribution of heterogeneous agents. The degree of welfare state protection (social insurance) is modelled as a level of consumption guaranteed to each agent in case of low realization of income. Thus the steady states differ only in this level of minimal guaranteed consumption: from almost no insurance at all ('low welfare regime') to a guaranteed consumption amounting to fifty percent of the average consumption in the economy ('high welfare regime').

The main result obtained from numerical simulations does not support the alleged efficiency-equality tradeoff attributed to welfare state economies. On the one hand, efficiency does fall monotonically as the social insurance provided by the government increases. While the efficiency loss is relatively small for the low and medium levels of social insurance, it grows rapidly to $17 \%$ of steady state's GDP in the case of the most generous welfare regime. On the other hand, equality improves only at the low and medium levels of minimal guaranteed consumption. When the level of social insurance becomes very high the equality measure worsens: the Gini coefficient equals 0.32 in the low welfare regime, it equals 0.23 in a welfare regime guaranteeing $36 \%$ of average consumption, and then it rises to 0.26 in the high welfare regime.

The main forces behind these efficiency and equality outcomes are the labor supply incentives and the general equilibrium effects of different market-clearing interest rates associated with each welfare regime. That a high level of guaranteed consumption is less efficient is natural since it is a constraint on optimal government policies. High levels of guaranteed consumption do not allow the government to use the low consumption levels as incentives to enforce high labor supply.

In equilibrium, different levels of social insurance imply different marketclearing prices and different distribution of resources. I will show that the average welfare increases in the level of social insurance while the welfare of median agents decreases. Second, the market-clearing interest rate declines in the level of mini- 
mal guaranteed consumption as the planners find it optimal to accumulate more capital in order to obtain higher output with a lower incentive compatible labor supply.

In order to evaluate how a reduction of social insurance affects the well-being of agents, I compute the expected discounted utility during transitions between the welfare regimes' steady states. These transitions can be interpreted as social insurance reforms recently considered and implemented in some European countries in order to improve economic performance. I find that a reduction of welfare regimes' generosity can be welfare improving if the efficiency gains to such a reform are redistributed back to the agents. These transfers are generally large enough to compensate the agents for increased labor supply and risk. In the following sections, I will analyze these efficiency, welfare, and distributional aspects in detail.

I will now describe the main features of the model. I consider an economy populated by a continuum of agents operating stochastic neoclassical technologies with capital and labor inputs. In order to insure against bad realizations of output, the risk-averse agents enter into insurance contracts with a zero profit insurance agency, modelled as a government (social planner). Since labor supply is private information of each agent, the government overcomes the moral hazard problem by conditioning each agent's insurance transfer on the entire history of his output realizations by rewarding high realizations of past and current output with high current, as well as future, consumption levels.

Finally, the government determines the minimal level under which each agent's consumption cannot fall. The extent of such a 'welfare regime' policy, together with the underlying private information, results in stationary equilibria with different feasible, constrained-optimal allocations and in invariant, non-degenerate distributions of resources in which the well-being of any individual depends on his idiosyncratic realization of output. The imposed minimal guaranteed consumption also serves as an exogenous lower bound on the utility entitlements needed to prevent a degenerate stationary distribution with no mobility. ${ }^{3}$

The model also incorporates general production technology and capital accumulation into the general equilibrium, dynamic private information economies 
with heterogeneous agents. I decentralize the economy using the concept of component planners developed by Atkeson and Lucas (1995). The component planners minimize the cost of resources needed to provide incentive compatible insurance contracts to agents with particular utility entitlements. In order to provide an efficient amount of capital input in agents' production technologies, the component planners trade one period capital loans at a market-clearing interest rate. The market for these loans is organized by a 'capital planner' who serves as a financial intermediary for the component planners. The capital stock is accumulated from the surpluses the component planners deposit with the capital planner in each period. Previous models with private information and capital accumulation have studied only special cases with restrictions on preferences and technology (as in Khan and Ravikumar (1997a) or Khan and Ravikumar (1997b)). In my decentralized economy with capital planner, not only are all variables endogenous but there are also no special restrictions on preferences, production technology, information structure, or contracts. Aiyagari and Williamson (1999) model a private information endowment economy where capital is not used in production of the agents and is not part of the principal-agent contract.

Finally, this decentralized capital market is also used for finding the endogenous interest rate in the stationary equilibrium. As in Kehoe and Levine (1993), private information lowers the equilibrium interest rate below the discount rate of the agents. Further, the higher the social insurance the more the interest rate decreases in order to satisfy the aggregate feasibility condition. Numerical simulation shows that the general equilibrium effects associated with capital accumulation are very important for the steady state allocations and the transition process.

This paper is an application of a theoretical model of optimal accumulation and distribution of capital in an economy with heterogeneous agents and moral hazard in Bohacek (2001). All analytical claims and mathematical proofs can be found there. It builds on the seminal contributions by Atkeson and Lucas (1992) and Atkeson and Lucas (1995), and the partial equilibrium models of Green (1987), Spear and Srivastava (1987), Atkeson (1991), and especially Phelan and Townsend (1991). The paper is organized as follows. In the next Section, I describe 
preferences, production technology, private information environment, and outline a social planner's problem. In Section 3, I decentralize the economy and define a stationary recursive equilibrium. Model parameterization and characterization of optimal insurance policies are presented in Section 4. I state and interpret the results of numerical simulations in Section 5. Section 6 concludes.

\section{The Economy}

In this section, I formally describe the economy, and state the assumptions on preferences and production function, commodity space, and information structure.

The closed economy is populated by a continuum of agents on unit interval. Time is discrete, $t=0,1,2, \ldots$. At time zero, all agents hold initial endowment of capital stock $k_{0}>0$. In each period all agents are endowed with one unit of time that can be divided into labor, $l_{t}$, and leisure, $1-l_{t}$. At the beginning of a period $t \geq 0$, an agent chooses labor supply, $l_{t}$, to be supplied together with capital stock, $k_{t}$, in his production technology $f\left(k_{t}, l_{t}\right)$. At the end of the period, he divides the realized output, $y_{t}$, into consumption, $c_{t}$, and investment towards the next period capital stock, $k_{t+1}$. The capital stock depreciates each period at a rate $\delta \in(0,1)$.

The production technology $f: \mathbb{R}_{+} \times[0,1] \rightarrow \mathbb{R}_{+}$is homogeneous of degree one, strictly increasing and strictly concave and continuously differentiable in both arguments. Further, I assume that for any inputs $k>0$ and $l>0, f(k, 0)=$ $f(0, l)=0$ and $\lim _{k \rightarrow 0} f_{k}(k, l)=\infty$ and $\lim _{k \rightarrow \infty} F_{k}(k, l)=0$.

This assumption implies that for any fixed labor supply, the graph of $f(\cdot, l)$ crosses the $\delta k$ line at some finite, maximal sustainable capital stock in a steady state. Denote $\bar{k}$ as the solution to the equation $\delta k=f(k, 1)$ when the labor supply is maximal. Finally, I impose non-negativity of capital holdings as a borrowing constraint on the agents so that $k \in K=[0, \bar{k}]$. It follows that feasible steady-state sets for output and consumption are also bounded, $Y=[0, \bar{y}]$ and $C=[0, \bar{c}]$, and all sets are compact.

In each period the agent derives a separable utility from consumption and leisure, $u\left(c_{t}\right)+v\left(1-l_{t}\right)$, discounted over time at $\beta \in(0,1)$. The functions $u$ : 
$[0, \bar{c}] \rightarrow \mathbb{R}$ and $v:[0,1] \rightarrow \mathbb{R}$ are twice continuously differentiable, strictly concave, strictly increasing in $c$ and decreasing in $l$, and bounded from above and below.

Suppose now that the production technology is stochastic. Specifically, the realization of output is subject to iid productivity shocks so that any output level $y \in(0, \bar{y}]$ can occur when positive inputs were used in the production technology. Formally, an agent's labor and capital inputs are related to output realizations by an exogenous probability measure $P$ on Borel measurable space $(Y, \mathcal{B}(Y))$ where $\mathcal{B}(Y)$ denotes Borelians on $Y$. The number $P(A \mid k, l)$ represents the probability of output $y$ being an element of $A \in \mathcal{B}(Y)$ given the inputs $k \in K$ and $l \in L$ were employed in the production function.

Assumption 1 For each $(k, l) \in(0, \bar{k}] \times(0,1]$, the probability $P(A \mid k, l)$ is strictly positive for all $A \in \mathcal{B}(Y)$.

The output realizations drawn from this stochastic technology are independent across agents and time periods. Except for the monotone likelihood ratio condition there are no other restrictions on the probability distribution known to all agents in the economy.

The problem of each agent is to maximize an expected, discounted lifetime utility subject to the budget constraint. Due to the stochastic technology, the risk-averse agents would like to write insurance contracts in order to smooth their consumption. Suppose, however, that the labor effort is not observed by other agents in the economy. I will model the insurance contracts as a cost-minimizing principal-agent problem in a closed economy with heterogeneous agents and moral hazard. In exchange for the insurance scheme, the planner in the model confiscates the capital stock of the agents and controls all the variables that are observable. It is assumed that there is no private storage and that the principal (social planner) can prevent each agent from trading with other agents.

Finally, in order to model social insurance policies, I assume that the social planner specifies a minimal consumption level $\underline{c}>0$ under which each agent's consumption cannot fall in any period of time. ${ }^{4}$

The social planner writes an insurance contract with each agent in the economy 
in terms of allocation sequence specifying his or her input and consumption in each period $t \geq 0$. This allocation sequence must satisfy the promise keeping and incentive compatibility constraints. For the former, the planner identifies each agent by an initial entitlement to expected, discounted utility $w_{0} \in W=[\underline{w}, \bar{w}] \subset$ $\mathbb{R}$ and history of output realizations. ${ }^{5}$ All agents identified with the same $w_{0}$ and the same history receive the same treatment. Let $\lambda_{0}$ denote the initial (arbitrary) distribution of expected utility entitlements on $(W, \mathcal{B}(W))$. I will interpret $\lambda_{0}(A)$ as a fraction of the population entitled to expected discounted utility in $A \in \mathcal{B}(W)$.

An efficient social planner will minimize the cost of the insurance scheme. The participation constraint requires the planner to deliver the expected discounted utility $w_{0}$ to the agent entitled to $w_{0}$. The incentive compatibility constraint ensures that each agent prefers the recommended labor supply to any labor supply deviation in each period of time. The aggregate feasibility constraints require that in each period the social planner divides the accumulated aggregate capital stock into capital input assignments for all agents $w_{0} \in W$, and that all goods produced but not consumed by the agents are added by the social planner to the depreciated current capital stock.

It is standard in the literature (see Atkeson and Lucas (1995) or Phelan and Townsend (1991)) to show that the sequential planning problem is equivalent to a recursive formulation with a utility entitlement $w \in W$ as a state variable for each agent. The utility entitlement $w$ summarizes the history of output realizations of each agent at the beginning of each period. In the next Section I will study a steady state of a closed economy described above in which the distribution of utility entitlements $\lambda$ will be time invariant and the levels of aggregate variables constant and feasible. Details of the sequential formulation can be found in Bohacek (2001). 


\section{A Decentralized Economy in Stationary Re- cursive Equilibrium}

Following Atkeson and Lucas (1995), the problem of finding efficient allocations can be solved by a partial decentralization with prices and "component planners" each responsible for allocating resources only to agents entitled to a utility entitlement $w$. Each component planner chooses an allocation that attains the utility entitlement of his subpopulation in such a way as to minimize the cost of attaining $w$ evaluated at prices determined in the market for capital goods traded by the component planners. All component planners must guarantee the common minimal consumption level associated with each welfare regime, $\underline{c}>0$.

The component planners borrow and lend capital according to the willingness of their agents to supply labor effort. One can imagine the capital trading intermediated by a zero-profit financial intermediary called 'capital planner'. The capital planner manages the accumulated aggregate capital stock which he lends to the component planners as capital input in each period at a constant market clearing interest rate $r$. Thus in the steady state, the economy exhibits a constant aggregate capital stock $\bar{K}_{r}$, a corresponding constant interest rate $r$, time invariant optimal policy rules of all component planners, and a time invariant distribution of utility entitlements $\lambda_{r}$, that satisfy the market-clearing conditions for a closed economy.

An agent $w$ is assigned inputs $k(w)$ and $l(w)$ as functions of the current utility entitlement, while consumption $c(w, y) \geq \underline{c}$ and the continuation utility entitlement $w^{\prime}(w, y)$, the next-period state variable, are also functions of current output realization. At the end of each period, each component planner repays the capital loan $(r+\delta) k(w)$ and deposits all remaining surplus $y-c(w, y)$ with the capital planner. In the steady state, the market clearing interest rate must be such that all the capital stock is lent out and the aggregate surplus exactly equals the depreciated capital stock $\delta \bar{K}_{r}$.

For a constant interest rate $r$, define an allocation policy of a component planner associated with subpopulation $w \in W$ as $\sigma_{r} \equiv\left\{k(w), l(w), c(w, y), w^{\prime}(w, y)\right\}$ 
where $k: W \rightarrow[0, \bar{k}], l: W \rightarrow[0,1], c: W \times Y \rightarrow[\underline{c}, \bar{c}]$, and $w^{\prime}: W \times Y \rightarrow \mathbb{R}$. The objective of each component planner is to minimize the present value of resources evaluated at the intertemporal price of resources $1 /(1+r)$ subject to the promise keeping, incentive compatibility, and the minimal guaranteed consumption constraints. For all $w \in W$ define a value function $V_{r}: W \rightarrow \mathbb{R}$ for the component planning problem and an operator $T_{r}$ on the space of bounded, continuous functions $D(W)$ as

$$
\begin{aligned}
& \left(T_{r} V_{r}\right)(w)= \\
& \inf _{\sigma_{r}} \int_{Y}\left\{c(w, y)+(r+\delta) k(w)-y+\frac{1}{1+r} V_{r}\left(w^{\prime}(w, y)\right)\right\} P(d y \mid k(w), l(w)),
\end{aligned}
$$

subject to the promise keeping constraint,

$$
w=\int_{Y}\left\{u(c(w, y))+v(1-l(w))+\beta w^{\prime}(w, y)\right\} P(d y \mid k(w), l(w))
$$

the incentive constraint,

$$
l(w) \in \arg \max _{\hat{l} \in L} \int_{Y}\left\{u(c(w, y))+v(1-\hat{l})+\beta w^{\prime}(w, y)\right\} P(d y \mid k(w), \hat{l}),
$$

and the minimal guaranteed consumption constraint,

$$
c(w, y) \geq \underline{c}
$$

specified for each welfare regime.

In a stationary recursive equilibrium, the distribution of utility entitlements is invariant. Denote a transition function $F_{r}: W \times \mathcal{B}(W) \rightarrow[0,1]$ where $F_{r}(w, A)$ represents a probability that an agent with a current utility entitlement $w \in W$ will be entitled to a next period $w^{\prime}$ that belongs to $A \in \mathcal{B}(W)$. It is now possible to state the definition of a stationary recursive equilibrium.

Definition 1 A stationary recursive equilibrium for a decentralized economy with welfare regime guaranteeing minimal consumption $\underline{c}$ is a constant interest rate $r$, a value function $V_{r}$, an allocation policy $\sigma_{r}$, a probability measure $\lambda_{r}$, and a law of motion for aggregate capital stock $\bar{K}_{r}$, such that 
1. at interest rate $r$, for all $w \in W$, the allocation policy $\sigma_{r}$ minimizes the objective function of each component planner (1) subject to the promise keeping constraint (2), the incentive constraint (3), and the welfare regime constraint (4);

2. the probability measure $\lambda_{r} \in \Lambda(W, \mathcal{B}(W))$ is invariant,

$$
\lambda_{r}(A)=\int_{W} F_{r}(w, A) \lambda_{r}(d w) \quad \text { for all } A \in \mathcal{B}(W)
$$

3. the aggregate capital stock is constant and finite,

$$
\bar{K}_{r} \equiv \int_{W} k(w) \lambda_{r}(d w)<\infty
$$

4. and the aggregate feasibility condition holds,

$$
\int_{W \times Y}\{y-c(w, y)\} P(d y \mid k(w), l(w)) \lambda_{r}(d w)=\delta \bar{K}_{r} .
$$

It follows from Theorems 9.2 in Stokey, Lucas, and Prescott (1989) that at a constant interest rate, the optimal allocations of the recursive and sequential formulations are equivalent. It is straightforward to apply the First Welfare Theorem as in Atkeson and Lucas (1992) in order to establish the efficiency outcome of the component planning problem.

\section{Optimal Policies and Numerical Simulation}

In this Section, I specify the parameters for preferences and production technology and define the welfare regimes. Detailed analysis related to existence, uniqueness as well as the properties of optimal allocations and the stationary distribution of utility entitlements can be found in Bohacek (2001).

Each agent who in any given period consumes resources $c$ and works $l$ units of time obtains flow utility $(1-\phi) \ln c+\phi \ln (1-l)$, with coefficients $\phi=2 / 3$ and a time discount parameter $\rho=0.1$ so that $\beta=\frac{1}{1+\rho}=0.9091$. The set of 
$Y=[0, \bar{y}]=[0, f(\bar{k}, \bar{l})]$ is determined by a Cobb-Douglas production function with $\alpha=0.36$. The capital stock depreciates at $\delta=0.10$.

At the market clearing interest rate $r$ the capital input is assigned according to the efficient capital-labor ratio for each level of recommended labor supply. Thus the realization of a particular output level is determined by a fixed, exogenous probability $P(y \mid l)$ specified for thirty one discrete levels of labor supply $l \in L=$ $0,0.025,0.05, \ldots, 0.75$, and two levels of output, $\underline{y}=0$ and $\bar{y}=f(\bar{k}, \bar{l})$. I assume that the low output is realized with probability $\operatorname{Prob}(y=\underline{y} \mid l)=1-l$, and vice versa for high output, $\operatorname{Prob}(y=\bar{y} \mid l)=l$. In sum, agents who supply more labor use proportionally more capital input and have a higher probability of realizing high output.

A welfare regime is defined by the level of minimal consumption guaranteed to each agent for all realizations of output. The minimal guaranteed consumption levels are set at $\underline{c}=0.01,0.025,0.05, \ldots, 0.25,0.275$. It was not possible to guarantee minimal consumption beyond 0.275 because for such high levels a feasible stationary equilibrium could not be simulated: The planners were not able to enforce incentive compatible labor supply that would produce output needed for consumption and investment feasible in a closed economy.

The exogenous lower bound on utility entitlements in each welfare regime corresponds to $\underline{w} \equiv(1-\beta)^{-1}\{u(\underline{c})+v(1)\}$, i.e., the discounted present value of receiving certain assignments of the minimal guaranteed consumption and full leisure. It is straightforward to show that an agent cannot be asked to supply any labor effort without consumption incentives and that $\underline{w}$ must be the lowest utility entitlement. In Aiyagari and Alvarez (1995) terminology, 'misery is not attainable' because the agent can always enjoy leisure. Obviously, higher minimal guaranteed level of consumption $\underline{c}$ implies a higher lower bound on utility entitlements. Correspondingly, define the upper bound as $\bar{w} \equiv(1-\beta)^{-1}\{u(\bar{c})+v(1)\}$.

In general, models with moral hazard exhibit non-convexity of the constraint set for the component planner's problem. This property is regained by randomization by lotteries as in Prescott and Townsend (1984) or Phelan and Townsend (1991). I show in Bohacek (2001) that consumption and the continuation util- 
ity entitlement are non-decreasing functions of the current utility entitlement and output realization, a standard result in the private information literature. I also establish that in each welfare regime the upper bound on utility entitlements is endogenous at $w^{*}<\bar{w}$. In other words, the planners cannot punish the agents below $\underline{w}$ and it is not cost efficient to reward them beyond the endogenous upper bound.

However, I prove that the upper bound is endogenous only for interest rate $r<\rho$ where the planners are more patient than the agents. For $r \geq \rho$ the distribution is degenerate and the stationary equilibrium is not feasible in a closed economy. The intuition is that if the component planners discount the future more than the agents, they distribute their costs relatively more into the future by increasing agents' continuation utility entitlements. In the limit, agents with the lowest utility entitlement $\underline{w}$ stay there while all the others amass at the exogenous upper bound $\bar{w}$. If $r=\rho$, there are many ergodic sets at utility levels where labor supply is zero. Such allocations are not feasible in a steady state because at such utility levels all agents consume but none works. The analysis confirms the results of Kehoe and Levine (1993), Huggett (1997) and Lucas (1992) that in a private information economy the market clearing interest rate is less than the agents' time preference parameter.

In a non-degenerate stationary equilibrium with mobility, planners associated with relatively poor agents have a surplus while those associated with wealthy agents have a deficit. The feasibility condition for a closed economy requires that the distribution of agents over all utility entitlements be such that the aggregate surplus is zero. In Bohacek (2001) it is proven that a higher interest rate leads to an invariant distribution with more mass on higher utility entitlements, and finally, that the aggregate deficit is an increasing function of the interest rate. Then the algorithm for finding the stationary recursive equilibrium iterates on interest rate: 1. Guess an initial interest rate; 2. Find the optimal policies for all component planners and the invariant distribution of utility entitlements; 3 . If the component planners spend more on consumption and investment than the agents produce, repeat with a lower interest rate (and vice versa for a surplus). 


\section{$5 \quad$ Results}

In this Section I present the results of numerical simulations. I compare the welfare regimes in terms of efficiency and equality as well as steady state levels of consumption, labor supply, and utility. Finally, I evaluate the cost and welfare implications of welfare state reforms that reduce the generosity of social insurance.

\subsection{Efficiency and Equality in the Welfare Regimes}

Unfortunately, a direct comparison of individual welfare regimes' efficiency is not possible: All welfare regimes are closed economies with efficient allocations subject to the imposed information and welfare regime constraints. Moreover, their market clearing interest rates and ergodic sets are different. Finally, a comparison with full-information economy is not possible because of the latter's degenerate stationary distribution.

In order to compare the welfare regimes' efficiency, I follow Atkeson and Lucas (1995) and adopt their definition of efficiency in the following experiment. A welfare regime is efficient if it attains a given distribution of utility entitlements $\lambda$ with inflow of outside resources $\tau$ and there is no other welfare regime that attains the same distribution of utility entitlements with inflow of outside resources $\hat{\tau}<\tau$. I calculate the present value of inflow of outside resources to each welfare regime on a transition from its initial steady state to a steady state of a chosen benchmark welfare regime. If $\tau<0$, the transition to the benchmark welfare regime is efficient: the government attains the original distribution of utility entitlements while being able to export resources during the transition process.

I choose the low welfare regime as the benchmark economy and simulate transitions from the steady states of all welfare regimes to its steady state. This approach can be interpreted as if all other welfare regimes undertook a reform of their social insurance policies, namely, reduced the minimal guaranteed consumption to that of the low welfare regime while honoring the original distribution of agents' utility entitlements. Assuming that the reforming economy is small relative to the 'world' (i.e., low) welfare regime economy, the interest rate will be the same as 
in the low welfare regime economy from the first period of transition. Therefore, the allocations of a component planner associated with agents entitled to $w$ will be the same as those of a component planner associated with the same agents in the low welfare regime. However, the distribution of utility entitlements will be changing: initiated from the invariant distribution of the original steady state, it will evolve according to the continuation utility entitlements policies of the low welfare regime to the latter steady state's stationary distribution.

To summarize, at the constant market-clearing interest rate $r_{L}$, the component planners of the reforming regime use the low welfare regime allocation policies $\sigma_{r_{L}}$ during all $t \geq 0$ periods of transition until the reforming economy converges to the steady state of the low welfare regime. During the transition the economy is not in equilibrium and the inflow of outside resources in each period will not be zero. The aggregate capital stock accumulated in the original steady state, $\bar{K}_{S S}$, is rented each period to the capital market at $\left(r_{L}+\delta\right)$ and it is maintained over time at its initial level while capital input is rented at the same price in each period. Therefore, the income from the initial aggregate stock of capital is $r_{L} \bar{K}_{S S}$ in each transition period. Bohacek (2001) proves that the initial stationary distribution of the reforming regime $\lambda_{r_{S}}$ will converge to the stationary distribution of the low welfare regime $\lambda_{r_{L}}$ in a finite number of periods.

The criterion for the efficiency comparison is the discounted present value of the inflow of outside resources into the economy in transition,

$$
\begin{aligned}
\tau_{S S \rightarrow L}=\sum_{t=0}^{\infty}\left(\frac{1}{1+r_{L}}\right)^{t} \int_{W \times Y}\left\{c_{L}(w, y)+\left(r_{L}+\delta\right) k_{L}(w)-y-r_{L} \bar{K}_{S S}\right\} \\
\cdot P\left(d y \mid k_{L}(w), l_{L}(w)\right) \lambda_{r_{L}, t}(d w) .
\end{aligned}
$$

If $\tau_{S S \rightarrow L}$ is negative, the original steady state of the reforming welfare regime was less efficient than that of the low welfare regime because it required more outside resources to attain the same distribution of utility entitlements. In other words, in this case the planners save resources during the transition and can export goods out of the reforming economy.

Table 2 summarizes the main results for all welfare regimes. I identify each welfare regime by a ratio of the minimal guaranteed consumption to the steady 
state average consumption (the first column) and use the following labels: a "low welfare regime" corresponds to a welfare regime with this ratio equal to 0.01 , the "medium welfare regime" to that of 0.36 , and a "high welfare regime" to that of 0.48 .

The second column shows the efficiency performance of each welfare regime in terms of expected per-period inflows of outside resources during transition to the low welfare regime as a percentage of the original welfare regime's steady state GDP. The efficiency loss is relatively small (less than 1\% of GDP) in regimes that guarantee minimal consumption up to $26 \%$ of their average steady state consumption. However, for the most generous welfare regimes the efficiency loss rises to more than $17 \%{ }^{6}$ The efficiency loss grows as the increasing minimal guaranteed consumption tightens the constraint imposed on planners' allocations. Necessarily, they become less efficient because they cannot enforce high labor effort without the incentive tools provided by low consumption levels and low continuation utility entitlements. The concavity of utility and production functions implies the increasingly poor efficiency performance of the more generous welfare regimes.

The steady state allocations are feasible through the general equilibrium effect related to the market-clearing interest rate. As in Lucas (1992) and Kehoe and Levine (1993), private information with moral hazard lowers the market-clearing interest rate below the discount rate of the agents. Moreover, the higher the guaranteed minimal consumption the more the equilibrium interest rate declines in order to satisfy the market clearing condition. For example, the interest rate in the low welfare regime is 0.092 while that of the medium welfare regime is 0.081 . The welfare regimes with more generous social insurance accumulate larger capital stock and exhibit greater capital/output and especially capital/labor ratios. Thus the relatively inefficient economies provide for similar amounts of steady state aggregate consumption and output as the more efficient ones. From the point of view of the planners, this is optimal for obtaining greater output with lower incentive compatible labor supply. However, maintaining such a large stock of capital in the steady state is also costly.

The third column of Table 2 displays the Gini coefficient of inequality in terms 
of consumption equivalents. The main result is that inequality is not monotonically decreasing in the degree of social insurance. The Gini coefficient decreases from 0.33 in the low welfare regime to 0.23 in the medium welfare regime guaranteeing $36 \%$ of average consumption. After that it increases to 0.26 in the high welfare regime.

Figure 1 summarizes the efficiency-equality tradeoff for the welfare regimes: It exists only for the low-to-medium welfare regimes where equality improves with increased social insurance. If social insurance increases beyond the medium welfare regime the inequality measure starts to grow. This outcome seems to be robust for various specifications of the stochastic production function I have used in numerical simulations. The inflection point of the Gini measures occurred between 0.30 and 0.38 of the ratio of minimal guaranteed to average consumption levels.

This result reflects the importance of incentives associated with the minimal guaranteed consumption levels on distribution of resources in individual welfare regimes. In the low welfare regime steady state there is a sizeable fraction of poor, hard working agents but also a large number of agents amassed at the high utility entitlements. The poor agents work hard with a high probability of becoming better off in the future. As social insurance increases, the planners cannot enforce high labor effort and the fraction of wealthy agents declines because high consumption rewards are not optimal for a lower labor effort. As a result, the Gini coefficient of inequality decreases from the low to the medium welfare regime. Finally, in the most generous welfare regimes inequality grows because almost all agents are concentrated near the lower bound on utility entitlements defined by the minimal guaranteed consumption while some agents are wealthy due to idiosyncratic luck in their productivity outcomes. ${ }^{7}$

To evaluate whether the welfare regimes reduce or eliminate poverty, I compare the economies in their relative poverty measures, both in terms of consumption equivalent units. The fourth column in Table 2 shows the proportion of the population below $50 \%$ of the median consumption equivalent (the so called head-count relative poverty measure, see Mitchell (1991)). The fifth column offers the poverty gap measured as the percentage of the aggregate consumption equivalent needed 
to be redistributed to agents below $50 \%$ of the median to bring them to this level.

Both measures have shapes similar to that of the Gini coefficients. The most effective poverty reduction occurs in the welfare regime guaranteeing $22 \%$ of average consumption (18\% of population in poverty and a poverty gap 1.7\%). Note that the high welfare regime is the worst in terms of the head-count poverty measure: the distribution is so concentrated near the minimal guaranteed consumption level that $36 \%$ agents live in relative poverty. On the other hand, the low welfare regime needs more goods to bring its agents to the poverty line: the absolute difference between the poor and the rest of the society is greater.

If one interprets the Swedish welfare system as being in its most generous steady state during the 1980s, the model simulations correspond to the Swedish data. Lindbeck (1997) documents that in the case of Sweden the distribution of income widened in the 1980 s by $3-5$ points in the Gini coefficient. There was also a rise in the fraction of households below the poverty line, if poverty is defined in terms of relative incomes. Lindbeck (1997) attributes this growth of inequality to Swedish welfare policies' negative incentive effect on education, labor supply and labor market participation.

The last two columns of Table 2 present the steady state levels of average and median welfare, measured in terms of the consumption equivalent. It is apparent that the properties of stationary distribution associated with individual welfare regimes make the average welfare and the welfare of the median agent very different: the average agent works more and consumes less in the low welfare state, and vice versa for the median agent. Thus the average welfare increases in social insurance while it falls for the median agent. Note also that the magnitudes are much higher for the median agent: his well-being in the high welfare regime is only $75 \%$ of that in the low welfare state since he consumes $10 \%$ more but has to supply $25 \%$ more labor effort. On the other hand, average welfare differs maximally $6 \%$ between any of the welfare regimes.

The distributional effects can be illustrated by the steady state shares of total consumption and labor supply of the bottom and top quintiles of agents. In the low welfare regime, the bottom quintile consumes $11 \%$ of total consumption and 
supplies $44 \%$ of labor (which is only $4 \%$ of total consumption equivalent). The high welfare regime significantly improves the situation of the poorest agents: their shares improve to $13 \%, 23 \%$, and $9 \%$, respectively. On the other hand, the top quintile in the low welfare regime consumes $26 \%$ of total consumption and supplies $5 \%$ of labor (corresponds to $34 \%$ of total consumption equivalent); in the high welfare regime these numbers equal $28 \%, 11 \%$, and $36 \%$, respectively.

These numbers reveal that the greatest impact of social insurance is on labor supply incentives: the planners associated with agents in the bottom quintile (i.e., the hardest working agents) in the high welfare regime enforce only half of the share of total labor supply as the planners in the low welfare regime. Note that although the share of labor supply of the wealthiest agents increases in the high welfare regime, their overall welfare is not much affected by the level of social insurance.

\subsection{Welfare State Reform}

Given the efficiency-equality relationship, a very important question is whether a transition to a more efficient welfare regime steady state could also make the agents better off. To do so, I evaluate two social insurance reforms: First, transitions from all welfare regime steady states to the steady state of the low welfare regime (guaranteeing 0.01 of average consumption). Second, transitions to the most equal (and still relatively efficient) medium welfare regime steady state (guaranteeing 0.36 of the average consumption). Welfare comparisons are based on average expected discounted per-period consumption equivalent during the transition as a percentage of the average consumption in the initial steady state.

For each social insurance reform I compare the following two scenarios: in the first one the per-period efficiency loss/gain from the reform is not transferred to the agents while in the second one it is. The comparison of these two scenarios is illustrative from the point of view of their different results and policy implications. In the first scenario (without transfers) neither the government nor the agents care how the social insurance reform is financed. It can be interpreted as a transition of 
a small open economy whose component planners are insignificant relative to the world's component planners and the feasibility constraint does not hold. In the second scenario (with transfers) the agents bear the full cost/benefit of the reform and the feasibility condition is satisfied because the component planners transfer the efficiency loss/gain to the agents. Thus the economy is small only in terms of prices (the interest rate). The drawback of the latter scenario is the fact that the component planners enforce the new incentive compatible allocations as if the agents did not receive the efficiency transfers.

Table 3 shows that it is crucial whether the agents face the full cost of the reform. In case of the reform that reduces social insurance to that of the low welfare regime (second and third columns) the agents are worse off if they do not receive the efficiency gain: reduction of social insurance increases the risk and creates a sizeable fraction of poor agents. However, the efficiency gains to the reform are so large that if they are redistributed among agents the outcome is reversed. For example, if the medium welfare regime reduces its social insurance, welfare increases by $2.8 \%$ if the agents receive the efficiency gain. The efficiency transfers make a huge difference $(22 \%)$ for the agents transiting from the most generous welfare state while the welfare improvement is relatively small (around one percent or less) in the less generous welfare regimes.

In general, simulation of welfare state reform reveals that the agents are better off if they internalize the efficiency gains. If the government can commit to returning the resources gained from making the economy more efficient, a reduction of social insurance would be on average welfare improving. Vice versa, without transfers a reduction of social insurance lowers average welfare because it only reduces the insurance scheme. If the government does not return the efficiency gain to the agents, the latter would always prefer the most generous welfare system. The last two columns in Table 3 show that a reform that increases social insurance makes the agents better off if they do not bear the cost of the efficiency loss caused by the reform.

To relate the simulation results to current policy issues faced by many European countries - a partial reduction of an overgrown welfare state system-, consider the 
fourth and fifth columns in Table 3. The last three lines depict transition from the most generous welfare regimes to the medium welfare regime associated with the lowest inequality and relatively small efficiency loss (3\%). Without the efficiency transfers the average welfare still decreases but only by less than one percent (column 4). However, if only a small part of the efficiency gain were distributed among the agents, the same reform would improve efficiency, equality, as well as average welfare in the economy (column 5).

An example of transition allocations during the just described welfare reform which replaces the social insurance guaranteeing $45 \%$ of average consumption with the medium welfare regime (guaranteeing 36\%) is shown in Figure 2. The top panel displays the time path of the inflow of outside resources, the middle panel the evolution of the Gini coefficient of inequality, and the last panel the time path of average consumption and labor supply (related in \% to their original levels). The relaxed constraint on the guaranteed minimal consumption allows the planners to enforce higher labor supply (dotted line) which requires greater compensation from higher consumption and continuation utility entitlements. This reduces the inequality measure during the first periods of transition while the efficiency gain stays similar in all periods. The distribution of agents rapidly converges to its new steady state and with it all the aggregate variables.

Finally, note that this example of a transition is Pareto-efficient: the planners' cost is reduced while all individual agents' expected discounted utility entitlement is duly delivered regardless of whether the economy undertakes the transition. However, it is true that the distributional changes during the transition make the average utility different from its initial value.

\section{Conclusions}

This paper studies the existence of the welfare state tradeoff between efficiency and equality in a general equilibrium model with social insurance and moral hazard. The main result does not support the claim for the welfare state tradeoff between efficiency and equality. Efficiency falls and equality improves as social insurance 
increases from low to medium levels. However, this process stops at higher levels of social insurance where efficiency rapidly declines while inequality begins to rise. Numerical simulations show that the efficiency loss can be quite heavy for the most generous welfare regimes. The transition analysis provides evidence that agents would always prefer reduction in generosity of the welfare system if they receive the efficiency gains to the reform.

This feature has important consequences for evaluating welfare state reforms. For example, Freeman, Topel, and Swedenborg (1997) conclude that "much of Sweden's welfare state went beyond what was necessary to eliminate poverty" and that the solution to the Swedish crisis in the 1990's "necessitates contraction of the welfare state". This model supports their claim that "reductions in [unemployment insurance] programs will not seriously affect the income distribution." If the level of social insurance is very high the inequality measure might even improve.

Is there an optimal level of social insurance that would minimize inequality without worsening the efficiency of the economy too much? The answer depends on a society's priorities. The simulated economies point to a reasonable compromise between efficiency loss and inequality with a minimal guaranteed consumption between $25-35 \%$ of the average consumption. Such levels of social insurance balance the efficiency loss at around 1-3\% of GDP for about a $30 \%$ reduction of inequality and poverty compared to the least generous and most efficient welfare regime. Finally, reducing the most generous social insurance to this level seems to be a great improvement to economic efficiency as well. 


\section{References}

Aiyagari, R. S. and F. Alvarez (1995). Stationary efficient distributions with private information: A tale of kings and slaves. Manuscript.

Aiyagari, R. S. and S. D. Williamson (1999). Credit in a random matching model with private information. Review of Economic Dynamics (2), 36-64.

Alvarez, F. and M. Veracierto (1999). Labor market policies in an equilibrium search model. NBER Macroeconomic Annual (14), 265-304.

Atkeson, A. (1991). International lending with moral hazard and risk of repudiation. Econometrica 9, 1069-1089.

Atkeson, A. and R. E. Lucas, Jr. (1992). On efficient distribution with private information. Review of Economic Studies 59, 427-453.

Atkeson, A. and R. E. Lucas, Jr. (1995). Efficiency and equality in a simple model of efficient unemployment insurance. Journal of Economic Theory 66, 64-98.

Benabou, R. (2000). Unequal societies: Income distribution and the social contract. American Economic Review 90(1), 96-129.

Bohacek, R. (2001). Capital accumulation and distribution in an economy with moral hazard. CERGE-EI Working Paper Series.

European Commission (1997). Social Protection in the Member States of the European Union. Brussels: European Commission.

Flora, P. (Ed.) (1986). Growth to Limits: The Western European Welfare States since World War II (1 ed.). Berlin: De Gruyter.

Freeman, R. B., R. Topel, and B. Swedenborg (Eds.) (1997). The Welfare State in Transition: Reforming the Swedish Model (1 ed.). Chicago, USA: NBER and The University of Chicago Press.

Green, E. J. (1987). Lending and the smoothing of uninsurable income. In E. Prescott and N. Wallace (Eds.), Contractual Arrangements for Intertemporal Trade. Minneapolis: University of Minnesota Press.

Hassler, J., J. V. Rodriguez Mora, K. Storesletten, and F. Zilibotti (2001). The survival of the welfare state. CEPR Working Paper.

Huggett, M. (1997). The one-sector growth model with idiosyncratic shocks: Steady states and dynamics. Journal of Monetary Economics 39, 385-403.

Kehoe, T. J. and D. K. Levine (1993). Debt-constraint asset market. Review of Economic Studies 60, 865-888.

Khan, A. and B. Ravikumar (1997a). Enduring relationships in an economy with capital. Manuscript.

Khan, A. and B. Ravikumar (1997b). Growth and risk-sharing with private information. Manuscript. 
Lindbeck, A. (1997). The swedish experiment. Journal of Economic Literature 35(3), 1273-1319.

Lindbeck, A., S. Nyberg, and J. W. Weibull (1999). Social norms and economic incentives in the welfare state. Quarterly Journal of Economics 94 (1), 1-35.

Lucas, Jr., R. E. (1992). On efficiency and distribution. The Economic Journal (102), 233-247.

Mitchell, D. (1991). Income Transfers in Ten Welfare States (1 ed.). Brookfield, USA: Avebury.

Mortensen, D. T. and C. A. Pissarides (1999). New developments in models of search in the labour market. CEPR Discussion Paper (2053), 1-81.

OECD (1994). Jobs Study: Evidence and Explanations. OECD Publications, Paris: OECD.

OECD (1997). National Accounts: Detailed Tables. OECD Publications, Paris: OECD.

Phelan, C. (1995). Repeated moral hazard and one-sided commitment. Journal of Economic Theory 66, 488-506.

Phelan, C. and R. M. Townsend (1991). Computing multi-period, informationconstrained optima. Review of Economic Studies 58, 853-881.

Prescott, E. C. and R. M. Townsend (1984). Pareto optima and competitive equilibria with adverse selection and moral hazard. Econometrica 52(1), 21-45.

Sinn, H.-W. (1995). A theory of the welfare state. Scandinavian Journal of Economics $97(4), 495-526$.

Spear, E. and S. Srivastava (1987). On repeated moral hazard with discounting. Review of Economic Studies 55, 599-617.

Stokey, N. L., R. E. Lucas, Jr., and E. C. Prescott (1989). Recursive Methods in Economic Dynamics. Cambridge: Harvard University Press. 


\section{Notes}

1. For example, in 1993, the British Department of Social Security estimated that the number of people receiving at least one social security benefit totalled 46 million, which is nearly $80 \%$ of the population. Of the total income of households in the bottom quintile of the British population, $74 \%$ consists of benefits in cash and kind (net of tax). Or for another example, two-thirds of Swedish youth receives some means-tested benefit at least once before they reach age twenty seven (Lindbeck (1997)).

2. A study by the Swedish National Audit Bureau estimates cheating on benefits around $9 \%$ of the payments in the benefit system. When the replacement ratio in the sick-benefit system in Sweden increased to 90-100 percent, people doubled their days out of work due to alleged sickness to an average of 26 days per year. For details, see Lindbeck (1997), Lindbeck, Nyberg, and Weibull (1999), Hassler, Rodriguez Mora, Storesletten, and Zilibotti (2001), or Benabou (2000). For a comprehensive analysis of these issues in Sweden see Freeman, Topel, and Swedenborg (1997).

3. Atkeson and Lucas (1995) and Phelan (1995) prevent a degenerate distribution at the infinitely low utility level by not allowing the agents to forego future consumption or by a competition between planners, respectively.

4. Note that modelling social insurance by specifying minimal consumption is the same as if the planner specified minimal income: in the latter case, moral hazard would require the planner to solve for agent's consumption and use it as the insurance policy tool.

5. I will discuss in detail the values of $\{\underline{w}, \bar{w}\}$ in Section 4 .

6. Mortensen and Pissarides (1999) report efficiency loss of four percent if in the U.S. economy the replacement ratio and payroll tax increased to the European levels. Alvarez and Veracierto (1999) obtain similar changes in output measures in their model of equilibrium search with unions, firing taxes, and unemployment benefits.

7. Sinn (1995) obtains similar result for a model in which insurance induces individuals to increase risk taking.

8. Note that all individual agents, including the median, are indifferent of the reform. In each case an agent's promised utility entitlement $w$ is duly delivered. 
Measures of Social Protection in OECD Countries

\begin{tabular}{|c|c|c|c|}
\hline \multirow[b]{2}{*}{ Country } & \multicolumn{2}{|c|}{$\begin{array}{l}\text { Unemployment Benefits } \\
\text { as \% of Average Income }\end{array}$} & \multirow{2}{*}{$\begin{array}{c}\text { Transfers } \\
\text { to Households } \\
\text { as \% of GDP }\end{array}$} \\
\hline & Single Person & Household & \\
\hline Austria & 50 & 54 & 28 \\
\hline Belgium & 62 & 62 & 28 \\
\hline Denmark & 88 & 89 & 31 \\
\hline Finland & 70 & 70 & 36 \\
\hline France & 70 & 70 & 29 \\
\hline Germany & 45 & 50 & 27 \\
\hline Greece & 53 & 64 & 19 \\
\hline Ireland & 45 & 62 & 22 \\
\hline Italy & 55 & 65 & 26 \\
\hline Luxembourg & 85 & 89 & 28 \\
\hline Netherlands & 84 & 84 & 33 \\
\hline Portugal & 74 & 74 & 18 \\
\hline Spain & 84 & 84 & 23 \\
\hline Sweden & 96 & 96 & 40 \\
\hline Switzerland & 76 & 86 & 28 \\
\hline United Kingdom & 41 & 70 & 27 \\
\hline Australia & 34 & 60 & 13 \\
\hline Canada & 70 & 70 & 18 \\
\hline Japan & 30 & 30 & 12 \\
\hline New Zealand & 34 & 56 & 18 \\
\hline United States & 29 & 31 & 14 \\
\hline Average Europe & 68 & 73 & 28 \\
\hline Average non-Europe & 39 & 49 & 15 \\
\hline
\end{tabular}

Sources: OECD (1994), European Commission (1997) and OECD

(1997). Notes: Unemployment benefits as percentage of average previous earnings before tax in the first year of unemployment for a single person and household (single person with dependent spouse). Transfers to households which entail financial costs or loss of income due to: old age (37\%); sickness (25\%); invalidity and disability (9\%); survivors (9\%); family (7\%); unemployment (5\%); occupational accidents and disease (2\%); housing (2\%); maternity (1\%); placement, vocational guidance, and resettlement (1\%); miscellaneous (2\%).

Table 1: Measures of Social Protection in the OECD countries. 


\begin{tabular}{|c|c|c|c|c|c|c|}
\hline \multicolumn{7}{|c|}{ Steady State Statistics (\%) } \\
\hline \multirow{2}{*}{$\begin{array}{c}\text { Welfare } \\
\text { Regime }^{a}\end{array}$} & \multirow{2}{*}{$\begin{array}{l}\text { Efficiency } \\
\text { Loss }^{b}\end{array}$} & \multirow{2}{*}{$\begin{array}{c}\text { Inequality } \\
\left(\mathrm{Gini}^{c}\right)\end{array}$} & \multicolumn{2}{|c|}{ Relative Poverty Measures } & \multicolumn{2}{|c|}{ Welfare $^{f}$} \\
\hline & & & $\overline{\text { Head-count }}^{d}$ & $\overline{\text { Poverty gap }}^{e}$ & $\overline{\text { Average }}$ & Median \\
\hline 0.01 & 0.0 & 0.33 & 29.8 & 5.1 & 94.3 & 100.0 \\
\hline 0.04 & 0.1 & 0.32 & 27.8 & 4.2 & 95.3 & 94.7 \\
\hline 0.08 & 0.2 & 0.31 & 25.1 & 3.2 & 96.3 & 89.3 \\
\hline 0.12 & 0.3 & 0.29 & 22.0 & 2.3 & 97.2 & 84.5 \\
\hline 0.16 & 0.4 & 0.28 & 19.6 & 1.8 & 98.0 & 80.9 \\
\hline 0.22 & 0.5 & 0.26 & 18.3 & 1.7 & 98.6 & 78.6 \\
\hline 0.26 & 0.7 & 0.25 & 18.4 & 1.8 & 98.9 & 77.3 \\
\hline 0.31 & 1.3 & 0.24 & 20.1 & 2.0 & 99.0 & 76.5 \\
\hline 0.36 & 2.8 & 0.23 & 22.8 & 2.3 & 99.1 & 76.0 \\
\hline 0.41 & 5.7 & 0.24 & 26.4 & 2.7 & 99.2 & 75.5 \\
\hline 0.45 & 10.7 & 0.25 & 30.1 & 3.1 & 99.5 & 75.2 \\
\hline 0.48 & 17.3 & 0.26 & 36.1 & 3.6 & 100.0 & 74.8 \\
\hline
\end{tabular}

Notes: Definition of welfare regimes in terms of guaranteed consumption to average consumption: low (0.01), medium (0.36), high (0.48). ${ }^{a}$ Guaranteed consumption to average consumption. ${ }^{b}$ Per period inflow of outside resources transfers during transition as $\%$ of GDP in the initial steady state. ${ }^{c}$ Gini coefficient of per-period expected consumption equivalent. ${ }^{d}$ Proportion of the population below a $50 \%$ of median consumption equivalent. ${ }^{e}$ Percentage of aggregate consumption equivalent redistributed to agents below $50 \%$ of median consumption equivalent to bring their consumption equivalent to this level. ${ }^{f}$ Average welfare and welfare of the median agent in terms of per-period expected consumption equivalent.

Table 2: Steady state statistics for the welfare regimes. 


\begin{tabular}{lccccc}
\hline \hline \multicolumn{4}{c}{ Average Welfare in Transition (in \%) } \\
\hline From & \multicolumn{3}{c}{ Transition to: } \\
Welfare & Low Welfare Regime & Medium Welfare Regime \\
\cline { 2 - 3 } \cline { 5 - 6 } Regime & No Transfers & Transfers & & No Transfers & Transfers \\
\hline 0.01 & $100.0^{*}$ & $100.0^{*}$ & & 113.8 & 92.2 \\
0.04 & 100.0 & 100.1 & 111.2 & 92.5 \\
0.08 & 100.0 & 100.3 & 108.6 & 93.0 \\
0.12 & 100.0 & 100.3 & 106.1 & 93.7 \\
0.16 & 99.9 & 100.4 & 104.0 & 94.5 \\
0.22 & 99.8 & 100.4 & 102.4 & 95.3 \\
0.26 & 99.6 & 100.6 & 101.2 & 96.3 \\
0.31 & 99.3 & 101.2 & 100.5 & 97.6 \\
0.36 & 98.8 & 102.9 & $100.0^{*}$ & $100.0^{*}$ \\
0.41 & 98.2 & 106.7 & 99.6 & 104.3 \\
0.45 & 97.5 & 113.4 & 99.4 & 111.4 \\
0.48 & 96.6 & 122.6 & 99.3 & 120.8 \\
\hline \hline
\end{tabular}

Notes: Definition of welfare regimes in terms of guaranteed consumption to average consumption: low (0.01), medium (0.36). Star denotes the welfare regime to which other regimes transit. 'No Transfers' denotes transition without transfers; 'Transfers' denotes transition with transfers. Welfare measured in terms of expected discounted per-period consumption equivalent during transition as percentage of expected consumption in the initial steady state.

Table 3: Average welfare in transition. 
Efficiency of welfare regimes (\%)
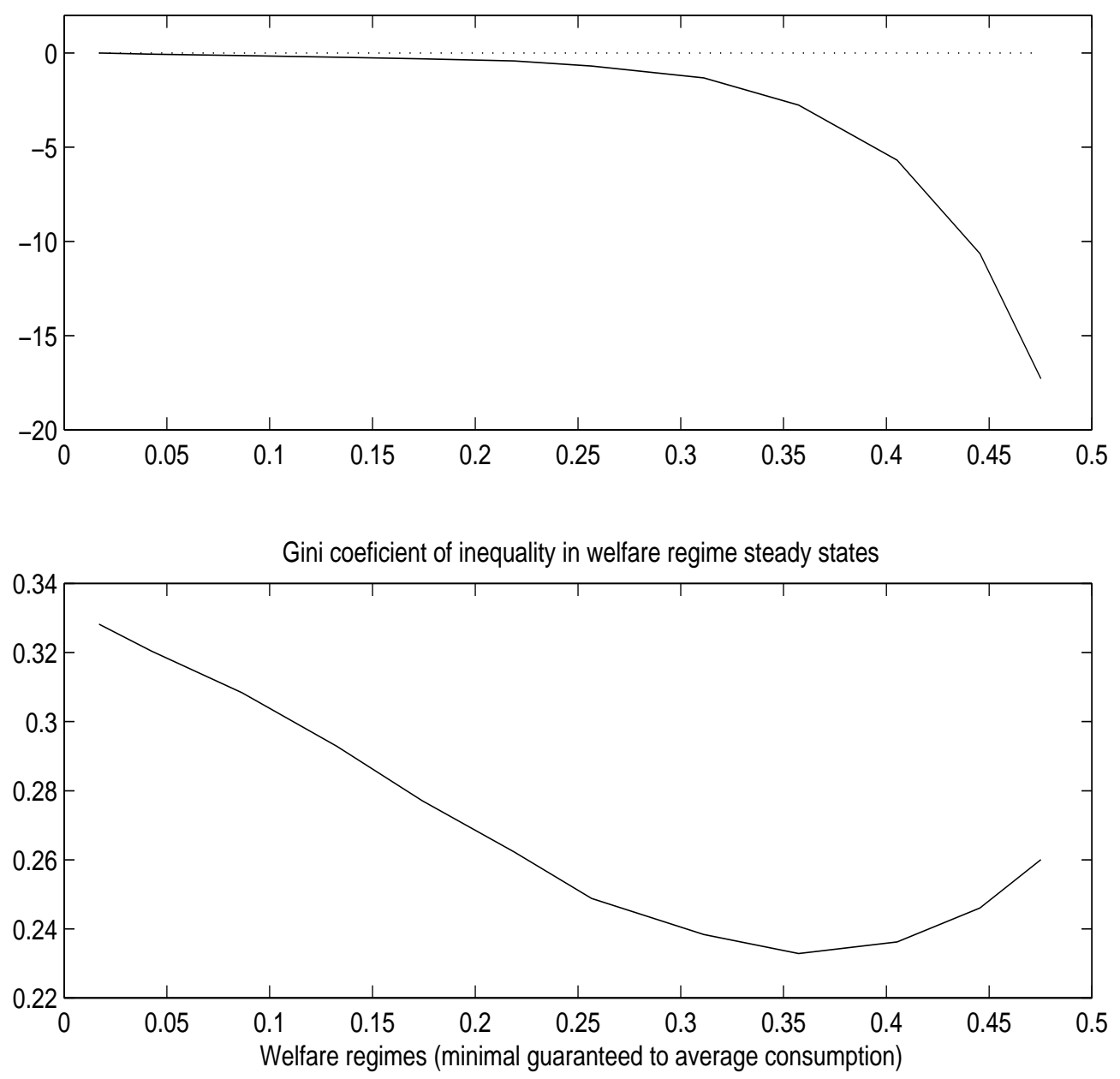

Figure 1: Equality and efficiency in welfare regime steady states. 

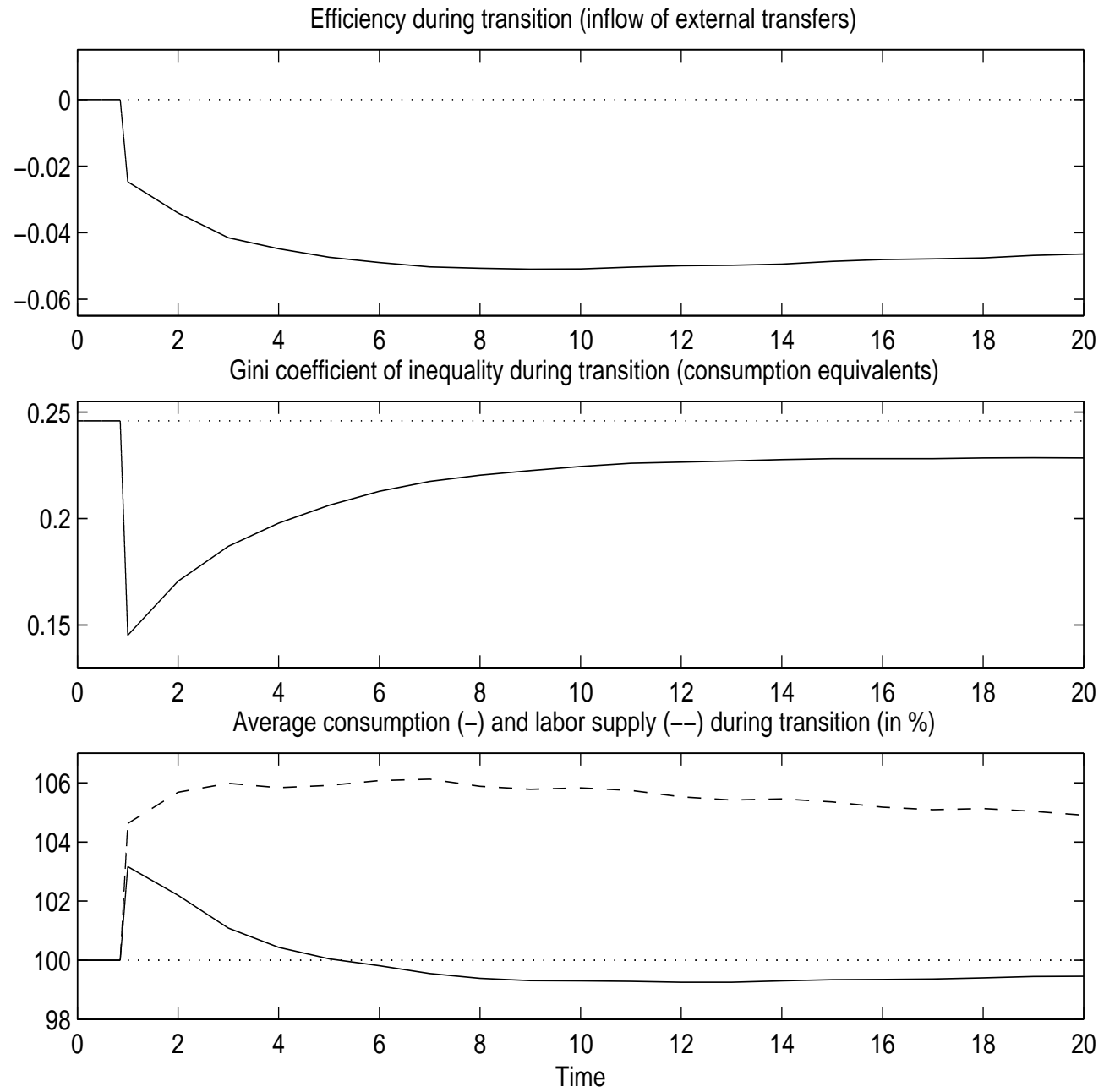

Figure 2: Efficiency, equality, and average consumption and labor supply during transition (without transfers) from a welfare regime guaranteeing 0.45 of average consumption to the medium (0.36) welfare regime. Reform starts in period one. 